\title{
Utilization and Safety of Percutaneous Lung Biopsy: A 10-Year Nationwide Population-Based Study
}

\author{
Chun-Ku Chen ${ }^{1,2,3,4}$, Hsiao-Ting Chang ${ }^{5,6}$, Yu-Chun Chen ${ }^{4,5,6} \oplus$, Shu-Chiung Chiang ${ }^{4}$, \\ Hsiao-Ping Chou ${ }^{1,2,7}$ and Tzeng-Ji Chen $4,5,6, * \mathbb{D}$ \\ 1 Department of Radiology, Taipei Veterans General Hospital, Taipei 11217, Taiwan; \\ ckchen@vghtpe.gov.tw (C.-K.C.); gutmomo@gmail.com (H.-P.C.) \\ 2 Department of Radiology, Faculty of Medicine, School of Medicine, National Yang-Ming University, \\ Taipei 11221, Taiwan \\ 3 Institute of Clinical Medicine, School of Medicine, National Yang-Ming University, Taipei 11221, Taiwan \\ 4 Institute of Hospital and Health Care Administration, School of Medicine, National Yang-Ming University, \\ Taipei 11221, Taiwan; yuchn.chen@gmail.com (Y.-C.C.); scchiang0g@gmail.com (S.-C.C.) \\ 5 Department of Family Medicine, Taipei Veterans General Hospital, Taipei 11217, Taiwan; \\ htchang2@vghtpe.gov.tw \\ 6 Department of Family Medicine, Faculty of Medicine, School of Medicine, National Yang-Ming University, \\ Taipei 11221, Taiwan \\ 7 Division of Radiology, Yonghe Cardinal Tien Hospital, New Taipei City 23445, Taiwan \\ * Correspondence: tjchen@vghtpe.gov.tw; Tel.: +886-2-28757458
}

Received: 9 March 2019; Accepted: 10 April 2019; Published: 12 April 2019

check for updates

\begin{abstract}
Percutaneous lung biopsy is a technique used for sampling peripherally located lung masses and has been gaining in popularity. However, its exact utilization is unknown, and its safety has not been well studied. The current study aimed to assess the trend of utilization and study the safety of this procedure. Using the National Health Insurance Research Database, we retrospectively determined the total number of procedures that were performed on subjects older than 20 years between 2001 and 2010. We also estimated the rates of major complications, such as pneumothorax, requiring intercostal drainage. A total of 630 percutaneous biopsies were performed in 2001, while 3814 were performed in 2010, representing a 6.1-fold increase. The compound annual growth rate was $22.1 \%$. The number of hospitals that performed the procedure increased from 55 to 99. Pneumothorax requiring drainage occurred in $1.5 \%$ of the procedures. The factors associated with a higher complication rate included male gender, chronic obstructive pulmonary disease, rural hospital, and low-volume hospital. Percutaneous lung biopsies are a relatively safe procedure, and their performance has been rapidly increasing. The number of procedures performed by a hospital was associated with the complication rate.
\end{abstract}

Keywords: lung biopsy; procedure safety; utilization

\section{Introduction}

Since its introduction, percutaneous lung biopsy has become an important method of obtaining lung tissue to characterize focal lung diseases such as lung cancer [1]. This technique does not require bronchoscopy via the pharynx and may be better tolerated by some patients. Using a cutting needle, a percutaneous lung biopsy is able to yield a larger specimen than that by bronchoscopic biopsy, thus, providing better diagnostic accuracy [2].

When compared with percutaneous lung biopsy, the bronchosopic procedure needs a preprocedural local spray of anesthetic agents to the throat, and some waiting time for the sprayed agents to be effective; moreover, for lesions that are not adjacent to the large airway or for peripheral 
lung lesions, additional fluoroscopic guidance may be needed, which would take more time with the addition of the operation of fluoroscopy. Modern techniques, such as electromagnetic navigation, can navigate the bronchoscopy and the devices to biopsy the peripheral pulmonary nodules via virtual three-dimensional images; however, the need to attach the sensor and load images in the planning phase would take even more time [3]. Regarding cost, the electromagnetic navigational bronchoscopic biopsy for the lung nodule has a higher cost than computed tomography (CT)-guided lung biopsy [4]. Bronchoscopy is also known to cause hypoxemia [5]. While percutaneous lung biopsy has several advantages over bronchoscopic lung biopsy as mentioned earlier, nevertheless, due to the percutaneous nature of this approach, the pleura will be punctured and pneumothorax is more likely to occur with this approach when compared with bronchoscopic biopsies [4].

Due to the rapidly increasing need for the histological typing of lung tissue [6,7], the need for percutaneous lung biopsies may continue to increase due to its suitability for the subtyping and genotyping of lung cancer [8], and the increasing use of CT scans for percutaneous lung biopsy could lower the availability of CT scanners for other purposes [9]. As percutaneous lung biopsies are most commonly performed with CT guidance, understanding their utilization trends may provide a reference regarding resource and equipment allocation for hospital administrators. CT advancements have enabled the detection of smaller nodules $[10,11]$, which make percutaneous biopsies technically more difficult [12], nevertheless, lung cancers of a small size had good prognosis [13]. Personnel training would be important if the utilization of percutaneous lung biopsy rapidly increases.

In the current study, we aimed to evaluate the utilization trends of percutaneous lung biopsy and estimate its safety profile with the aim of identifying the associated risk factors for complications.

\section{Materials and Methods}

\subsection{Database}

This study used the National Health Insurance Research Database, which consists of de-identified secondary data derived from the claims and registry data of the Taiwan National Health Insurance (NHI) Program beginning in 1995. Under this program, the Bureau of National Health Insurance (BNHI) has enrolled 99.5\% of the nation's inhabitants [14,15]. Annually, the Taiwan National Health Research Institute (NHRI) de-identifies and publishes the registry and claims data released by the BNHI. Before publication, the NHRI replaces all information about the beneficiaries, surgeons, and institutions with random combinations of characters to protect their privacy. Through this method, the NHRI not only makes it feasible to identify claims related to the same beneficiary, surgeon, or institution but it also makes the identification of a specific beneficiary, surgeon, or institution impossible. This study was approved by the Institutional Review Board of Taipei Veterans General Hospital (VGHIRB No.2013-02-015BC). Informed consent was waived as the database is de-identified and specific individuals could not be identified.

\subsection{Identification of Percutaneous and Bronchoscopic Lung Biopsy Cases}

We identified all records with a code of percutaneous lung biopsy (ICD-9-CM procedure code 33.26) or bronchoscopic lung biopsy (ICD-9-CM procedure code 33.27) from the claims database between 1 January 2001 and 31 December 2009.

\subsection{Demographics and Comorbid Conditions}

The ages of the subjects at the time of biopsy were identified, and the insurance levels were used as a proxy for socioeconomic status. All of the subjects' income levels were grouped into three categories of premiums paid (NTD\$ $\geq 40,000,20,000-39,999,1-19,999$ ). In the Taiwan NHI, health insurance premiums are primarily determined based on the insured wage and premium rate, thus, a higher premium implies a higher income. 
The urbanization of the location of the NHI registration is recorded as a proxy of residential urbanization. Taiwan is divided into seven urbanization levels, with level 1 defined as the "most urbanized" and level 7 defined as the "least urbanized". We designated level 1 as the metropolitan area; levels $2-3$ as the urban area, and levels $4-7$ as the rural area.

We also identified the baseline comorbidities associated with a complicated lung biopsy as follows: chronic obstructive pulmonary disease (COPD) (ICD-9-CM code: 490-496.x), renal failure (530.0-530.9), chronic liver disease and cirrhosis (571.x), coagulation defects and hemorrhagic conditions (286-287.x), ischemic heart disease (410-414.x), and stroke (430.1x, 433.x1, 434.x, 436.x).

\subsection{Hospital Characteristics}

The hospitals performing the biopsies were identified as medical centers, regional hospitals, or local hospitals. The medical centers were tertiary referral centers, and because the volume of procedures performed outside of the medical centers was small, we combined regional and local hospitals into a non-medical center category. The number of percutaneous lung biopsies performed was divided by the median value and rounded to the nearest 100 biopsies per year. The urbanization of the hospital was designated by the same method as that used to designate residence urbanization, as described in the previous section.

\subsection{Outcomes}

Perioperative complications were defined as complications if they occurred within 30 days of the biopsy procedure. Pneumothorax requiring drainage was defined as a diagnostic code of pneumothorax (ICD-9-CM code: 512.x) and the insertion of a chest tube (ICD-9-CM procedure code: 34.04 ) in the same visit. Hemoptysis (ICD9-CM code 786.3), cardio-pulmonary resuscitation (ICD-9-CM procedure code: 99.60), surgery for hemorrhage control (ICD-9-CM procedure code: 39.98), and exploratory thoracotomy (ICD-9-CM procedure code: 34.02 ) were all searched.

\subsection{Statistical Analysis}

Data management and computing were performed with Microsoft SQL Server 2012 (Microsoft Corporation, Redmond, WA, USA). Statistical analysis was performed with SPSS (Version 20.0, IBM, Armonk, NY, USA). The compound annual growth rate (CAGR) was calculated to represent the trend of utilization by the following equation:

$$
\left.C A G R=\left(\left(N_{2010} / N_{2001}\right)^{\wedge} 1 / 9\right)\right)-1
$$

Complication rates were computed as the number of each event divided by the number of procedures performed during the study period. If a subject had more than one lung biopsy procedure, only the first procedure was included. We excluded any procedures associated with tuberculosis or pleurisy, as the insertion of an intercostal catheter is likely and could lead to an overestimation of the complications. Categorical variables were compared using the $\chi^{2}$ test or Fisher's exact test, as appropriate, and continuous variables were compared using the Student's $t$-test. To compare the complications, if there were multiple biopsies for a single subject, only the first biopsy was included. Associations between the hospital characteristics and the outcomes were assessed by multivariate logistic regression. The trends of percutaneous and bronchoscopic lung biopsies were analyzed by the $\chi^{2}$ test for linear trends. $\mathrm{P}<0.05$ (two-sided) was considered significant.

\section{Results}

\subsection{Overall Utilization}

A total of 20,518 percutaneous lung biopsy procedures were performed in 19,330 subjects. The total number of percutaneous lung biopsy procedures increased from 630 in 2001 to 3814 in 2010, 
and the number of hospitals performing percutaneous biopsies increased from 55 in 2001 to 99 in 2010. There was a 6.1-fold increase in percutaneous lung biopsies between 2001 and 2010. The CAGR for percutaneous lung biopsies was $22.1 \%$ during the same period. The number of bronchoscopic procedures was 1278 in 2001 and peaked in 2009 with 2252 procedures (but fell to 2040 in 2010). The number of hospitals performing bronchoscopic lung biopsies was 67 in 2001, with a slight increase to 90 in 2010 (Figure 1). The ratio of percutaneous to bronchoscopic lung biopsies showed an increasing trend from 2001 to 2010.

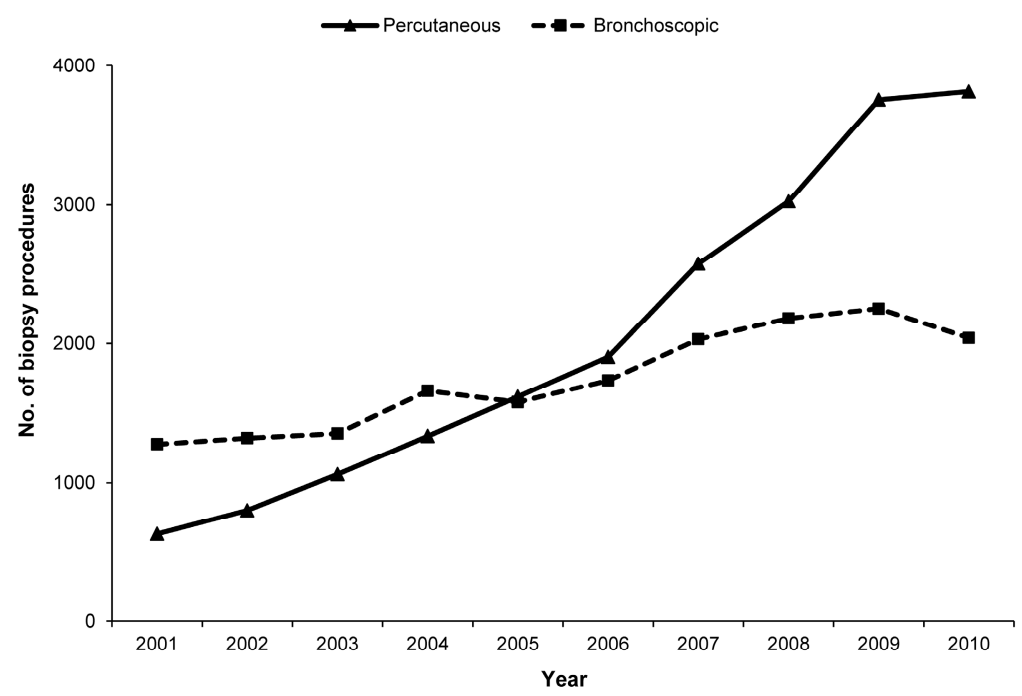

Figure 1. Trends in the utilization of lung biopsies. The use of percutaneous lung biopsies increased rapidly between 2001 and 2010 .

Percutaneous lung biopsies constituted 33\% and 65\% of all non-surgical lung biopsies in 2001 and 2010, respectively (Figure 2). Significant differences were found in the trends of utilization between percutaneous lung biopsy and bronchoscopic biopsy $(p<0.001)$.

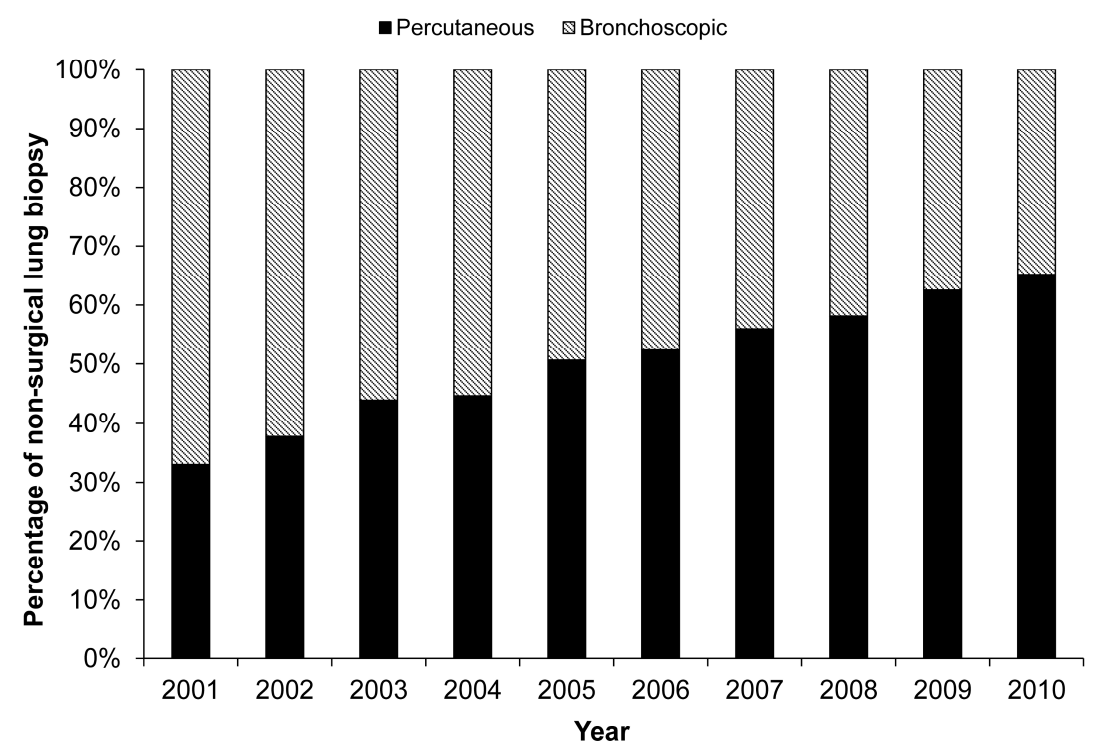

Figure 2. Trends in the ratio of percutaneous lung biopsies to non-surgical lung biopsies. In 2001, only $33 \%$ of the lung biopsies were percutaneous, whereas in 2010, percutaneous biopsies constituted $65 \%$ of all lung biopsies. 


\subsection{Utilization Based on Hospital Characteristics}

Approximately $38 \%$ of the percutaneous lung biopsies were performed in the Taipei metropolitan region, whereas only $24 \%$ of the bronchoscopic lung biopsies were performed in the same area. Northern Taiwan hospitals performed only $7 \%$ of the percutaneous lung biopsies, but performed $23 \%$ of the bronchoscopic lung biopsies. The ratio of percutaneous to bronchoscopic biopsies was close to 1:1 in the Central, South, and Kaohsiung Metropolitan areas (Table 1).

Table 1. Utilization and hospital characteristics.

\begin{tabular}{lcccccccccccc}
\hline \multirow{2}{*}{ Hospital Characteristics } & \multicolumn{10}{c}{ Year } & Total \\
\cline { 2 - 9 } & $\mathbf{2 0 0 1}$ & $\mathbf{2 0 0 2}$ & $\mathbf{2 0 0 3}$ & $\mathbf{2 0 0 4}$ & $\mathbf{2 0 0 5}$ & $\mathbf{2 0 0 6}$ & $\mathbf{2 0 0 7}$ & $\mathbf{2 0 0 8}$ & $\mathbf{2 0 0 9}$ & $\mathbf{2 0 1 0}$ & \\
\hline Total No. & 630 & 802 & 1061 & 1337 & 1616 & 1902 & 2576 & 3026 & 3754 & 3814 & 20,518 \\
$\quad \begin{array}{l}\text { Hospital characteristics } \\
\quad \text { Urbanization }\end{array}$ & & & & & & & & & & & \\
$\quad$ Metropolitan & 314 & 385 & 481 & 607 & 663 & 724 & 928 & 898 & 1163 & 1192 & 7355 \\
$\quad$ Urban & 257 & 343 & 484 & 573 & 676 & 881 & 1349 & 1846 & 2280 & 2271 & 10,960 \\
$\quad$ Rural & 59 & 74 & 96 & 157 & 277 & 297 & 299 & 282 & 311 & 351 & 2203 \\
$\quad$ Geographical Region & & & & & & & & & & & \\
$\quad$ Taipei Metropolitan & 270 & 300 & 365 & 517 & 540 & 693 & 923 & 1153 & 1473 & 1516 & 7750 \\
$\quad$ Northern & 93 & 109 & 130 & 171 & 214 & 144 & 189 & 128 & 189 & 163 & 1530 \\
$\quad$ Central & 32 & 66 & 115 & 142 & 186 & 247 & 402 & 551 & 679 & 698 & 3118 \\
$\quad$ Kaohsiung & 112 & 179 & 265 & 322 & 415 & 462 & 528 & 580 & 691 & 716 & 4270 \\
Metropolitan & & & & & & & & & & & \\
$\quad$ Southern & 86 & 96 & 128 & 146 & 193 & 319 & 449 & 515 & 618 & 606 & 3156 \\
$\quad$ Eastern & 37 & 52 & 58 & 39 & 68 & 37 & 85 & 99 & 104 & 115 & 694 \\
$\quad$ Referral status & & & & & & & & & & & \\
$\quad$ Medical Center & 445 & 587 & 774 & 918 & 1019 & 1157 & 1684 & 1957 & 2414 & 2398 & 13,353 \\
$\quad$ Non-medical center & 185 & 215 & 287 & 419 & 597 & 745 & 892 & 1069 & 1340 & 1416 & 7165 \\
\hline
\end{tabular}

Medical centers (tertiary referral centers) performed 445 biopsies in 2001 and 2398 in 2010, whereas non-medical centers performed 185 in 2001 and 1416 in 2010. The CAGRs for medical centers and regional/local hospitals were 20.6 and $25.4 \%$, respectively. There was a difference in the trends of percutaneous lung biopsy utilization between the different hospital levels $(p<0.001)$.

The use of percutaneous lung biopsy procedures differed between hospital urbanization categories, with CAGRs of $16.0 \%, 27.4 \%$, and $21.9 \%$ for metropolitan, urban, and rural hospitals, respectively.

\subsection{Complications}

Among the 19,330 subjects who underwent percutaneous lung biopsies, 1112 subjects were associated with tuberculosis or pleurisy and were excluded from the analysis of complications. From the total of 18,218 percutaneous lung biopsies that were included in the analysis, the rate of intercostal drainage catheter use due to pneumothorax was $1.5 \%$. There was a $0.1 \%$ overall exploratory thoracotomy rate and a $0.2 \%$ overall cardiopulmonary resuscitation rate (Table 2). Nevertheless, major complication-free outcomes (no cardiopulmonary resuscitation, no exploratory thoracotomy, no chest tube insertion for pneumothorax, no operation for hemorrhage) were noted in $98.1 \%$ of percutaneous lung biopsy procedures.

Table 2. Complications of percutaneous lung biopsy.

\begin{tabular}{lcc}
\hline Complications $(\boldsymbol{N}=\mathbf{1 8 , 2 1 8})$ & No. & $\mathbf{( \% )}$ \\
\hline Pneumothorax with drainage & 274 & 1.5 \\
Hemoptysis & 290 & 1.6 \\
Cardiopulmonary resuscitation & 41 & 0.2 \\
Exploratory thoracotomy & 17 & 0.1 \\
\hline
\end{tabular}


We further analyzed the factors associated with major complications (Table 3). Male subjects, subjects older than 65 years, subjects who underwent percutaneous lung biopsies in a hospital in a rural area, subjects with COPD, renal failure, and subjects who had undergone percutaneous biopsy at a low-volume hospital or non-medical center were more likely to have suffered a pneumothorax requiring intercostal drainage. Male subjects, elderly subjects, and subjects who received a biopsy in a rural, low-volume, or non-medical center hospital were more likely to suffer hemoptysis. Subjects who were male and elderly, had COPD, had lower income, had a stroke, or underwent procedures in a low-volume hospital or non-medical center hospital had higher cardiopulmonary resuscitation rates. Subjects who had coagulation disorders and who underwent percutaneous lung biopsies in a rural hospital or a non-medical center were more likely to have an exploratory thoracotomy.

Table 3. Subjects and hospital characteristics and complications.

\begin{tabular}{|c|c|c|c|c|c|c|c|c|c|c|c|c|c|}
\hline \multirow[t]{2}{*}{ Characteristics } & \multirow{2}{*}{$\begin{array}{c}\text { Total } \\
\text { No. }\end{array}$} & \multicolumn{3}{|c|}{$\begin{array}{l}\text { Pneumothorax } \\
\text { with Drainage }\end{array}$} & \multicolumn{3}{|c|}{ Hemoptysis } & \multicolumn{3}{|c|}{$\begin{array}{l}\text { Cardiopulmonary } \\
\text { Resuscitation }\end{array}$} & \multicolumn{3}{|c|}{$\begin{array}{l}\text { Exploratory } \\
\text { Thoracotomy }\end{array}$} \\
\hline & & $n$ & $(\%)$ & $p$-Value & $n$ & $(\%)$ & $p$-Value & $n$ & $(\%)$ & $p$-Value & $n$ & $(\%)$ & $p$-Value \\
\hline \multicolumn{14}{|l|}{ Demographics } \\
\hline Gender & & & & $<0.001$ & & & 0.008 & & & 0.03 & & & 0.27 \\
\hline Female & 6626 & 65 & $(1.0)$ & & 84 & (1.3) & & 8 & $(0.1)$ & & 4 & $(0.1)$ & \\
\hline Male & 11,592 & 209 & $(1.8)$ & & 206 & (1.8) & & 33 & $(0.3)$ & & 13 & $(0.1)$ & \\
\hline Age & & & & 0.006 & & & 0.008 & & & 0.003 & & & 0.70 \\
\hline$<65$ & 7728 & 94 & $(1.2)$ & & 145 & (1.9) & & 8 & $(0.1)$ & & 8 & $(0.1)$ & \\
\hline$\geq 65$ & 10,490 & 180 & (1.7) & & 145 & (1.4) & & 33 & $(0.3)$ & & 9 & $(0.1)$ & \\
\hline Insurance amount & & & & 0.15 & & & 0.29 & & & 0.02 & & & 0.68 \\
\hline$<20,000$ & 3890 & 48 & $(1.2)$ & & 57 & (1.5) & & 15 & $(0.4)$ & & 4 & $(0.1)$ & \\
\hline $20,000-39,999$ & 10,363 & 171 & (1.7) & & 178 & (1.7) & & 23 & $(0.2)$ & & 8 & $(0.1)$ & \\
\hline$\geq 40,000$ & 3965 & 55 & (1.4) & & 55 & (1.4) & & 3 & $(0.1)$ & & 5 & $(0.1)$ & \\
\hline Subject Urbanization & & & & 0.49 & & & 0.14 & & & 0.04 & & & 0.19 \\
\hline Metropolitan & 5057 & 71 & (1.4) & & 79 & (1.6) & & 11 & $(0.2)$ & & 5 & $(0.1)$ & \\
\hline Urban & 7796 & 127 & (1.6) & & 111 & (1.4) & & 11 & $(0.1)$ & & 4 & $(0.1)$ & \\
\hline Suburb/Rural & 5365 & 76 & (1.4) & & 100 & (1.9) & & 19 & $(0.4)$ & & 8 & $(0.1)$ & \\
\hline \multicolumn{14}{|l|}{ Comorbidities } \\
\hline Cirrhosis & & & & 0.91 & & & 0.30 & & & 0.05 & & & 0.21 \\
\hline No & 16,656 & 250 & (1.5) & & 270 & (1.6) & & 34 & $(0.2)$ & & 17 & $(0.1)$ & \\
\hline Yes & 1562 & 24 & (1.5) & & 20 & (1.3) & & 7 & $(0.4)$ & & 0 & $(0.0)$ & \\
\hline Coagulation disorders & & & & 0.61 & & & 0.81 & & & 0.13 & & & 0.03 \\
\hline No & 17,942 & 269 & (1.5) & & 287 & (1.6) & & 39 & $(0.2)$ & & 15 & $(0.1)$ & \\
\hline Yes & 276 & 5 & $(1.8)$ & & 3 & $(1.1)$ & & 2 & $(0.7)$ & & 2 & $(0.7)$ & \\
\hline COPD & & & & $<0.001$ & & & 0.873 & & & 0.02 & & & 0.12 \\
\hline No & 14,704 & 174 & $(1.2)$ & & 233 & (1.6) & & 27 & $(0.2)$ & & 11 & $(0.1)$ & \\
\hline Yes & 3514 & 100 & $(2.8)$ & & 57 & (1.6) & & 14 & $(0.4)$ & & 6 & $(0.2)$ & \\
\hline Diabetes Mellitus & & & & 0.05 & & & 0.05 & & & 0.34 & & & $>0.99$ \\
\hline No & 14,437 & 230 & (1.6) & & 243 & $(1.7)$ & & 30 & $(0.2)$ & & 14 & $(0.1)$ & \\
\hline Yes & 3781 & 44 & $(1.2)$ & & 47 & $(1.2)$ & & 11 & $(0.3)$ & & 3 & $(0.1)$ & \\
\hline Hypertension & & & & 0.87 & & & 0.16 & & & 0.98 & & & 0.11 \\
\hline No & 11,587 & 173 & (1.5) & & 196 & $(1.7)$ & & 26 & $(0.2)$ & & 14 & $(0.1)$ & \\
\hline Yes & 6631 & 101 & (1.5) & & 94 & (1.4) & & 15 & $(0.2)$ & & 3 & $(0.0)$ & \\
\hline Ischemic heart disease & & & & 0.17 & & & 0.14 & & & 0.47 & & & $>0.99$ \\
\hline No & 15,803 & 230 & (1.5) & & 260 & (1.6) & & 34 & $(0.2)$ & & 15 & $(0.1)$ & \\
\hline Yes & 2415 & 44 & (1.8) & & 30 & $(1.2)$ & & 7 & $(0.3)$ & & 2 & $(0.1)$ & \\
\hline Renal failure & & & & 0.02 & & & 0.11 & & & 0.50 & & & 0.62 \\
\hline No & 17,202 & 250 & (1.5) & & 280 & (1.6) & & 38 & $(0.2)$ & & 17 & $(0.1)$ & \\
\hline Yes & 1016 & 24 & $(2.4)$ & & 10 & $(1.0)$ & & 3 & $(0.3)$ & & 0 & $(0.0)$ & \\
\hline Stroke & & & & 0.72 & & & 0.04 & & & 0.02 & & & 0.63 \\
\hline No & 16,986 & 254 & (1.5) & & 279 & (1.6) & & 34 & $(0.2)$ & & 17 & $(0.1)$ & \\
\hline Yes & 1232 & 20 & (1.6) & & 11 & $(0.9)$ & & 7 & $(0.6)$ & & 0 & $(0.0)$ & \\
\hline
\end{tabular}


Table 3. Cont.

\begin{tabular}{|c|c|c|c|c|c|c|c|c|c|c|c|c|c|}
\hline \multirow[t]{2}{*}{ Characteristics } & \multirow{2}{*}{$\begin{array}{l}\text { Total } \\
\text { No. }\end{array}$} & \multicolumn{3}{|c|}{$\begin{array}{l}\text { Pneumothorax } \\
\text { with Drainage }\end{array}$} & \multicolumn{3}{|c|}{ Hemoptysis } & \multicolumn{3}{|c|}{$\begin{array}{c}\text { Cardiopulmonary } \\
\text { Resuscitation }\end{array}$} & \multicolumn{3}{|c|}{$\begin{array}{l}\text { Exploratory } \\
\text { Thoracotomy }\end{array}$} \\
\hline & & $n$ & $(\%)$ & $p$-Value & $n$ & $(\%)$ & $p$-Value & $n$ & $(\%)$ & $p$-Value & $n$ & $(\%)$ & $p$-Value \\
\hline \multicolumn{14}{|l|}{ Hospital characteristics } \\
\hline Hospital Urbanization & & & & 0.001 & & & $<0.001$ & & & 0.06 & & & 0.04 \\
\hline Metropolitan & 6567 & 77 & $(1.2)$ & & 98 & $(1.5)$ & & 13 & $(0.2)$ & & 4 & $(0.1)$ & \\
\hline Urban & 9739 & 152 & (1.6) & & 122 & (1.3) & & 19 & $(0.2)$ & & 8 & $(0.1)$ & \\
\hline Suburb/Rural & 1912 & 45 & (2.4) & & 70 & (3.7) & & 9 & $(0.5)$ & & 5 & $(0.3)$ & \\
\hline Volume (100) & & & & 0.007 & & & $<0.001$ & & & 0.04 & & & 0.02 \\
\hline Low & 10,862 & 185 & (1.7) & & 230 & $(2.1)$ & & 31 & $(0.3)$ & & 15 & $(0.1)$ & \\
\hline High & 7356 & 89 & (1.2) & & 60 & $(0.8)$ & & 10 & $(0.1)$ & & 2 & $(0.03)$ & \\
\hline Hospital grade & & & & 0.04 & & & $<0.001$ & & & $<0.001$ & & & 0.01 \\
\hline Medical center & 12,013 & 165 & (1.4) & & 130 & $(1.1)$ & & 15 & $(0.1)$ & & 6 & $(0.05)$ & \\
\hline Non-medical center & 6205 & 109 & (1.8) & & 160 & (2.6) & & 26 & (0.4) & & 11 & $(0.2)$ & \\
\hline
\end{tabular}

Bronchoscopic biopsies were associated with a $98.1 \%$ major complication-free rate, $0.3 \%$ cardiopulmonary resuscitation rate, $0.1 \%$ exploratory thoracotomy rate, and $1.1 \%$ rate of intercostal drainage for pneumothorax. We found no significant difference in the major complication-free rates between bronchoscopy and percutaneous lung biopsy $(p=0.829)$.

The results of the multivariate analysis are shown in Table 4. Male gender, COPD, rural hospital, and low-volume hospital were associated with pneumothorax requiring drainage. Male gender, elderly, rural hospital, low-volume hospital, and non-medical center were associated with hemoptysis. Non-medical centers were associated with cardiopulmonary resuscitation. Coagulation disorders were associated with exploratory thoracotomy.

Table 4. Multivariate analysis for factors associated with complications.

\begin{tabular}{|c|c|c|c|c|c|c|c|c|c|c|c|c|}
\hline \multirow[t]{2}{*}{ Characteristics } & \multicolumn{3}{|c|}{$\begin{array}{l}\text { Pneumothorax with } \\
\text { Drainage }\end{array}$} & \multicolumn{3}{|c|}{ Hemoptysis } & \multicolumn{3}{|c|}{$\begin{array}{l}\text { Cardiopulmonary } \\
\text { Resuscitation }\end{array}$} & \multicolumn{3}{|c|}{ Exploratory Thoracotomy } \\
\hline & $\begin{array}{l}\text { Odds } \\
\text { Ratio }\end{array}$ & $95 \%$ CI & $p$-Value & $\begin{array}{l}\text { Odds } \\
\text { Ratio }\end{array}$ & $95 \%$ CI & $p$-Value & $\begin{array}{l}\text { Odds } \\
\text { Ratio }\end{array}$ & $95 \%$ CI & $p$-Value & $\begin{array}{l}\text { Odds } \\
\text { Ratio }\end{array}$ & $95 \%$ CI & $p$-Value \\
\hline \multicolumn{13}{|l|}{ Demographics } \\
\hline Male gender & 1.56 & $(1.17-2.08)$ & 0.002 & 1.48 & $(1.14-1.92)$ & 0.003 & 1.99 & $(0.90-4.38)$ & 0.09 & 1.66 & $(0.53-5.24)$ & 0.39 \\
\hline Age $\geq 65$ & 1.18 & $(0.90-1.56)$ & 0.23 & 0.68 & $(0.53-0.88)$ & 0.003 & 2.17 & $(0.96-4.92)$ & 0.06 & 0.70 & $(0.24-2.00)$ & 0.50 \\
\hline Insurance amount & & & 0.27 & & & 0.30 & & & 0.09 & & & 0.52 \\
\hline$<20,000$ & 1.00 & (reference) & & 10.00 & (reference) & & 10.00 & (reference) & & 1.00 & (reference) & \\
\hline $20,000-39,999$ & 1.31 & $(0.94-1.81)$ & 0.11 & 1.18 & $(0.87-1.60)$ & 0.28 & 0.62 & $(0.32-1.20)$ & 0.15 & 0.78 & $(0.23-2.63)$ & 0.69 \\
\hline$\geq 40,000$ & 1.25 & $(0.83-1.86)$ & 0.29 & 0.95 & $(0.65-1.40)$ & 0.80 & 0.27 & $(0.08-0.97)$ & 0.04 & 1.56 & $(0.39-6.28)$ & 0.54 \\
\hline Subject Urbanization & & & 0.07 & & & 0.50 & & & 0.30 & & & 0.33 \\
\hline Rural & 1.00 & (reference) & & 10.00 & (reference) & & 10.00 & (reference) & & 1.00 & (reference) & \\
\hline Urban & 1.38 & $(0.97-1.97)$ & 0.08 & 1.12 & $(0.80-1.56)$ & 0.50 & 0.98 & $(0.43-2.23)$ & 0.95 & 0.93 & $(0.26-3.31)$ & 0.91 \\
\hline Metropolitan & 1.42 & $(1.05-1.92)$ & 0.02 & 0.94 & $(0.70-1.25)$ & 0.65 & 0.56 & $(0.26-1.22)$ & 0.14 & 0.41 & $(0.11-1.44)$ & 0.16 \\
\hline \multicolumn{13}{|l|}{ Comorbidities } \\
\hline Cirrhosis & 0.90 & $(0.59-1.39)$ & 0.65 & 0.76 & $(0.47-1.20)$ & 0.24 & 1.66 & $(0.71-3.85)$ & 0.24 & 0.00 & (0.00-Inf) & 0.99 \\
\hline Coagulation disorders & 1.12 & $(0.46-2.77)$ & 0.80 & 0.72 & $(0.23-2.27)$ & 0.57 & 3.03 & $(0.71-12.96)$ & 0.14 & 13.99 & $(3.05-64.08)$ & 0.001 \\
\hline COPD & 2.14 & $(1.64-2.81)$ & $<0.001$ & 1.03 & $(0.75-1.40)$ & 0.86 & 10.36 & $(00.69-20.71)$ & 0.38 & 2.42 & $(0.82-7.11)$ & 0.11 \\
\hline Diabetes Mellitus & 0.70 & $(0.50-0.98)$ & 0.04 & 0.77 & $(0.56-1.07)$ & 0.11 & 10.27 & $(00.62-20.61)$ & 0.52 & 1.11 & $(0.31-4.06)$ & 0.87 \\
\hline Hypertension & 0.93 & $(0.70-1.22)$ & 0.58 & 1.07 & $(0.81-1.40)$ & 0.64 & 00.70 & $(00.34-10.43)$ & 0.33 & 0.39 & $(0.10-1.50)$ & 0.17 \\
\hline Ischemic heart disease & 1.01 & $(0.71-1.43)$ & 0.96 & 0.88 & $(0.59-1.32)$ & 0.54 & 00.94 & $(00.39-20.24)$ & 0.88 & 1.26 & $(0.26-6.18)$ & 0.78 \\
\hline Renal failure & 1.40 & $(0.91-2.16)$ & 0.13 & 0.58 & $(0.31-1.11)$ & 0.10 & 00.86 & $(00.26-20.88)$ & 0.81 & 0.00 & $(0.00-$ Inf $)$ & 0.99 \\
\hline Stroke & 0.96 & $(0.60-1.54)$ & 0.86 & 0.56 & $(0.30-1.04)$ & 0.07 & 20.12 & $(00.89-50.06)$ & 0.09 & 0.00 & (0.00-Inf) & 0.99 \\
\hline \multicolumn{13}{|l|}{ Hospital characteristics } \\
\hline Hospital Urbanization & & & 0.002 & & & 0.001 & & & 0.67 & & & 0.71 \\
\hline Rural & 1.00 & (reference) & & 1.00 & (reference) & & 10.00 & (reference) & & 10.00 & (reference) & \\
\hline Urban & 0.43 & $(0.27-0.69)$ & $<0.001$ & 0.66 & $(0.44-0.98)$ & 0.04 & 10.35 & $(00.48-30.81)$ & 0.57 & 0.50 & $(0.09-2.71)$ & 0.42 \\
\hline Metropolitan & 0.57 & $(0.38-0.86)$ & 0.007 & 0.52 & $(0.37-0.74)$ & $<0.001$ & 00.96 & $(00.40-20.32)$ & 0.93 & 0.65 & $(0.18-2.37)$ & 0.51 \\
\hline
\end{tabular}


Table 4. Cont.

\begin{tabular}{|c|c|c|c|c|c|c|c|c|c|c|c|c|}
\hline \multirow[t]{2}{*}{ Characteristics } & \multicolumn{3}{|c|}{$\begin{array}{c}\text { Pneumothorax with } \\
\text { Drainage }\end{array}$} & \multicolumn{3}{|c|}{ Hemoptysis } & \multicolumn{3}{|c|}{$\begin{array}{l}\text { Cardiopulmonary } \\
\text { Resuscitation }\end{array}$} & \multicolumn{3}{|c|}{ Exploratory Thoracotomy } \\
\hline & $\begin{array}{l}\text { Odds } \\
\text { Ratio }\end{array}$ & $95 \% \mathrm{CI}$ & $p$-Value & $\begin{array}{l}\text { Odds } \\
\text { Ratio }\end{array}$ & $95 \%$ CI & $p$-Value & $\begin{array}{l}\text { Odds } \\
\text { Ratio }\end{array}$ & $95 \%$ CI & $p$-Value & $\begin{array}{l}\text { Odds } \\
\text { Ratio }\end{array}$ & $95 \% \mathrm{CI}$ & $p$-Value \\
\hline Volume & & & 0.04 & & & 0.001 & & & 0.47 & & & 0.16 \\
\hline Low & 1.00 & (reference) & & 1.00 & (reference) & & 10.00 & (reference) & & 10.00 & (reference) & \\
\hline High & 0.72 & $(0.52-0.98)$ & & 0.55 & $(0.39-0.78)$ & & 10.49 & $(00.50-40.42$ & & 0.29 & $(0.05-1.60)$ & \\
\hline Hospital grade & & & 0.15 & & & 0.007 & & & 0.01 & & & 0.50 \\
\hline Non-medical center & 1.00 & (reference) & & 1.00 & (reference) & & 1.00 & (reference) & & 10.00 & (reference) & \\
\hline Medical center & 1.29 & $(0.92-1.83)$ & & 0.64 & $(0.46-0.88)$ & & 0.26 & $(0.09-0.74)$ & & 0.63 & $(0.17-2.40)$ & \\
\hline
\end{tabular}

\section{Discussion}

The present study demonstrated a rapid increase in the utilization of percutaneous lung biopsies during 2001-2010. The complication rate observed was reasonable, and there were no differences in the major complication rates between percutaneous lung biopsies and bronchoscopic lung biopsies.

The use of bronchoscopic lung biopsies increased slowly from 2001 to 2009, but decreased in 2010, whereas the use of percutaneous lung biopsies increased rapidly throughout the same period. The observed increasing popularity of percutaneous lung biopsies is consistent with the findings of a previous study [16].

The previously reported rate of pneumothorax requiring drainage in percutaneous lung biopsies was 0 to $6.6 \%$ [17-19]. The current study showed that the rate of pneumothorax requiring drainage was $1.5 \%$, which is slightly lower than some previously reported values [18]. The fewer passages through the pleural surface may have decreased the probability of a significant pneumothorax. Moore et al. found that if only one pleural puncture were used, the chest tube placement rate was $0.4 \%$ when compared with an overall chest tube placement rate of 1.6\%. [20] However, Ohno et al. found that a single puncture resulted in no chest tube insertion, whereas three punctures in a lung resulted in a chest tube insertion rate of $50 \%$. Similarly, a study using a coaxial needle revealed no chest tube insertion rate in cases of pneumothorax [17]. Assuming that coaxial usage was routine in our study due to the fact that coaxial needles are reimbursed by the BNHI and no subjects had to pay out-of-pocket costs, the rate of pneumothorax requiring drainage was reasonably accurate.

The possible reason for the increased popularity of percutaneous lung biopsies was possibly due to the accuracy of percutaneous biopsies. A previous report found that percutaneous CT-guide biopsy had a diagnostic yield of $93 \%$, higher than that of bronchoscopy biopsy at $75 \%$. Additionally, the proportion of the more commonly regarded centrally located squamous cell carcinoma has decreased over the past 30 years, from $49.6 \%$ to $34.8 \%$ [21]. Moreover, traditionally recognized centrally located squamous cell carcinoma has been reported to consist of more than $62 \%$ of squamous cell carcinoma [22].

The strengths of our study include its use of a large, population-based sample that is representative of the national population of Taiwan, which was made possible by accessing data from a compulsory, government-run, centralized, single-payer universal health insurance system covering treatment for all major medical or surgical events. Healthcare services provided by overseas providers are also covered by the system. Loss of follow-up is, therefore, only possible if users do not seek medical attention, suggesting that our estimates of incidence are likely to be accurate and representative of the national population.

This study has several limitations. First, it is unclear whether pneumothorax requiring drainage is a complication of or related to percutaneous lung biopsy, as there were no medical charts available for review. Nevertheless, it is less likely that a biopsy would have been performed to investigate a pneumothorax when compared with the probability that a pneumothorax would be an unintentional complication of a biopsy. Moreover, we excluded the common reason, which is pleural effusion for intercostal drainage insertion. Thus, we considered this issue to be at least partially addressed. Second, the rate of pneumothorax may have been underestimated. Our standards required a concurrent diagnosis of pneumothorax in addition to the performance of an intercostal drainage procedure to 
qualify as a pneumothorax complication. However, these stipulations mean that minor cases of pneumothorax may have been missed.

\section{Conclusions}

In conclusion, percutaneous lung biopsy utilization increased rapidly between 2001 and 2010. This procedure is relatively safe, although the volume of procedures performed by a given hospital was associated with the complication rate.

Author Contributions: Conceptualization, C.-K.C., H.-T.C., and H.-P.C.; Data Curation, C.-K.C.; Formal Analysis, C.-K.C. and H.-T.C.; Methodology, C.-K.C., Y.-C.C., S.-C.C., and T.-J.C.; Project Administration, H.-P.C. and T.-J.C.; Resources, C.-K.C. and T.-J.C.; Supervision, H.-T.C. and T.-J.C.; Validation, Y.-C.C., S.-C.C., H.-P.C., and T.-J.C.; Writing-Original Draft, C.-K.C., H.-T.C., and H.-P.C.; Writing-Review and Editing, C.-K.C., Y.-C.C, and T.-J.C.

Funding: This research received no external funding.

Acknowledgments: This study was based partly on data from the NHRI database provided by the BNHI, Department of Health and managed by the NHRI in Taiwan. The interpretation and conclusions contained herein do not represent the opinion of the BNHI, the Department of Health, or the NHRI.

Conflicts of Interest: The authors declare no conflict of interest. The funders had no role in the design of the study, in the collection, analyses or interpretation of data, in the writing of the manuscript, and in the decision to publish the results.

\section{References}

1. Haaga, J.R.; Alfidi, R.J. Precise biopsy localization by computer tomography. Radiology 1976, 118, $603-607$. [CrossRef] [PubMed]

2. Kardos, L.; Nagy, E.; Morvay, Z.; Fuzesi, E.; Furak, J.; Tiszlavicz, L.; Horvath, I.; Palko, A. Value of CT-guided biopsy compared to fluoroscopy-guided transthoracic biopsy and bronchoscopic sampling in the diagnosis of pulmonary nodules. Orv. Hetil. 1999, 140, 931-933. [PubMed]

3. Bhatt, K.M.; Tandon, Y.K.; Graham, R.; Lau, C.T.; Lempel, J.K.; Azok, J.T.; Mazzone, P.J.; Schneider, E.; Obuchowski, N.A.; Bolen, M.A. Electromagnetic Navigational Bronchoscopy versus CT-guided Percutaneous Sampling of Peripheral Indeterminate Pulmonary Nodules: A Cohort Study. Radiology 2018, 286, 1052-1061. [CrossRef] [PubMed]

4. Dale, C.R.; Madtes, D.K.; Fan, V.S.; Gorden, J.A.; Veenstra, D.L. Navigational bronchoscopy with biopsy versus computed tomography-guided biopsy for the diagnosis of a solitary pulmonary nodule: A cost-consequences analysis. J. Bronchol. Interv. Pulmonol. 2012, 19, 294-303. [CrossRef] [PubMed]

5. Albertini, R.E.; Harrell, J.H.; Kurihara, N.; Moser, K.M. Arterial hypoxemia induced by fiberoptic bronchoscopy. JAMA 1974, 230, 1666-1667. [CrossRef] [PubMed]

6. Herbst, R.S.; Lippman, S.M. Molecular signatures of lung cancer-toward personalized therapy. N. Engl. J. Med. 2007, 356, 76-78. [CrossRef] [PubMed]

7. Herbst, R.S.; Heymach, J.V.; Lippman, S.M. Lung cancer. N. Engl. J. Med. 2008, 359, 1367-1380. [CrossRef] [PubMed]

8. Hsiao, S.H.; Chung, C.L.; Lee, C.M.; Chen, W.Y.; Chou, Y.T.; Wu, Z.H.; Chen, Y.C.; Lin, S.E. Suitability of computed tomography-guided biopsy specimens for subtyping and genotyping of non-small-cell lung cancer. Clin. Lung Cancer 2013, 14, 719-725. [CrossRef] [PubMed]

9. Brenner, D.J.; Hall, E.J. Computed tomography-An increasing source of radiation exposure. N. Engl. J. Med. 2007, 357, 2277-2784. [CrossRef] [PubMed]

10. Henschke, C.I.; McCauley, D.I.; Yankelevitz, D.F.; Naidich, D.P.; McGuinness, G.; Miettinen, O.S.; Libby, D.M.; Pasmantier, M.W.; Koizumi, J.; Altorki, N.K.; et al. Early Lung Cancer Action Project: Overall design and findings from baseline screening. Lancet 1999, 354, 99-105. [CrossRef]

11. New York Early Lung Cancer Action Project Investigators. CT Screening for lung cancer: Diagnoses resulting from the New York Early Lung Cancer Action Project. Radiology 2007, 243, 239-249.

12. Ng, Y.L.; Patsios, D.; Roberts, H.; Walsham, A.; Paul, N.S.; Chung, T.; Herman, S.; Weisbrod, G. CT-guided percutaneous fine-needle aspiration biopsy of pulmonary nodules measuring $10 \mathrm{~mm}$ or less. Clin. Radiol. 2008, 63, 272-277. [CrossRef] [PubMed] 
13. Wang, B.Y.; Hung, J.J.; Jeng, W.J.; Hsu, W.H.; Hsieh, C.C.; Huang, M.H.; Huang, B.S.; Liu, J.S.; Wu, Y.C. Surgical outcomes in resected non-small cell lung cancer $<$ or $=1 \mathrm{~cm}$ in diameter. J. Chin. Med. Assoc. 2010, 73, 308-313. [PubMed]

14. Social Indicators 2009. Directorate General of Budget, Accounting and Statistics. Available online: https: //ebook.dgbas.gov.tw/public/Data/331311345571.pdf (accessed on 8 March 2019).

15. National Health Insurance. The National Health Insurance Statistics 2009. Available online: https: //www.nhi.gov.tw/english/Content_List.aspx?n=A8FFBD35C8B89CEB\&topn=616B97F8DF2C3614 (accessed on 8 March 2019).

16. Tukey, M.H.; Wiener, R.S. Population-based estimates of transbronchial lung biopsy utilization and complications. Respir. Med. 2012, 106, 1559-1565. [CrossRef] [PubMed]

17. Yeow, K.M.; See, L.C.; Lui, K.W.; Lin, M.C.; Tsao, T.C.; Ng, K.F.; Liu, H.P. Risk factors for pneumothorax and bleeding after CT-guided percutaneous coaxial cutting needle biopsy of lung lesions. J. Vasc. Interv. Radiol. 2001, 12, 1305-1312. [CrossRef]

18. Wiener, R.S.; Schwartz, L.M.; Woloshin, S.; Welch, H.G. Population-based risk for complications after transthoracic needle lung biopsy of a pulmonary nodule: An analysis of discharge records. Ann. Intern. Med. 2011, 155, 137-144. [CrossRef] [PubMed]

19. Laurent, F.; Montaudon, M.; Latrabe, V.; Begueret, H. Percutaneous biopsy in lung cancer. Eur. J. Radiol. 2003, 45, 60-68. [CrossRef]

20. Moore, E.H.; Shepard, J.A.; McLoud, T.C.; Templeton, P.A.; Kosiuk, J.P. Positional precautions in needle aspiration lung biopsy. Radiology 1990, 175, 733-735. [CrossRef] [PubMed]

21. Tsukazan, M.T.R.; Vigo, A.; Silva, V.D.D.; Barrios, C.H.; Rios, J.O.; Pinto, J.A.F. Lung cancer: Changes in histology, gender, and age over the last 30 years in Brazil. J. Bras. Pneumol. 2017, 43, 363-367. [CrossRef] [PubMed]

22. Krimsky, W.; Muganlinskaya, N.; Sarkar, S.; Vulchi, M.; Patel, P.; Rao, S.; Hammer, J.; Evans, R.; Qureshi, M.; Harley, D. The changing anatomic position of squamous cell carcinoma of the lung-A new conundrum. J. Community Hosp. Int. Med. Perspect. 2016, 6, 33299. [CrossRef] [PubMed]

(C) 2019 by the authors. Licensee MDPI, Basel, Switzerland. This article is an open access article distributed under the terms and conditions of the Creative Commons Attribution (CC BY) license (http://creativecommons.org/licenses/by/4.0/). 